\title{
Network Selection with Imprecise Information in Heterogeneous All-IP Wireless Systems
}

\author{
Farooq Bari \\ AT\&T \\ $7277164^{\text {th }}$ Avenue NE \\ Redmond, WA 98052, USA \\ farooq.bari@att.com
}

\author{
Victor Leung \\ Dept. of Electrical \& Computer Engineering \\ The University of British Columbia \\ Vancouver, BC, Canada V6T $1 Z 4$ \\ vleung@ece.ubc.ca
}

\begin{abstract}
Selection of an optimal service delivery network is an important problem to solve in an all IP heterogeneous wireless access network environment. Several network parameters impact the process of network selection in such an environment and ideally their precise values should be known by the decision maker. In reality, however, the exact values for many of the parameters, e.g., those related to quality of service, will not be known. Hence there is a need to develop a network selection mechanism for scenarios when some of the parameter values are less reliable or unavailable. This paper describes a novel and comprehensive network selection approach that combines parameter estimation techniques with fuzzy theory and multi attribute decision making algorithm to perform network selection. In addition the paper proposes a new concept of Confidence Level in the network rankings that leverages additional available information in the final decision process. The proposed techniques provide improved network selection in heterogeneous all IP wireless access environment.
\end{abstract}

\section{Categories and Subject Descriptors}

C.2.1 [Computer-Communication Networks]: Network Architecture and Design - Wireless communication, Packetswitching networks.

\section{General Terms}

Algorithms.

\section{Keywords}

Network selection, Heterogeneous wireless systems, Multi Attribute Decision Making (MADM).

\section{INTRODUCTION}

Broadband wireless networks such as wireless local area networks (WLANs) and wireless wide area networks (WWANs) commonly employ Internet Protocol (IP) at the network layer. In order to

Permission to make digital or hard copies of all or part of this work for personal or classroom use is granted without fee provided that copies are not made or distributed for profit or commercial advantage and that copies bear this notice and the full citation on the first page. To copy otherwise, to republish, to post on servers or to redistribute to lists, requires prior specific permission and/or a fee. WICON 2007, October 22-24, Austin, USA

Copyright (C) 2007 978-963-9799-12-7

DOI 10.4108/wicon.2007.2093 provide ubiquitous coverage, increasingly these all IP wireless technologies are being made to inter-work. This makes the consistent service experience over heterogeneous wireless technologies very important. Unlike circuit switched service environments, packet switched IP networks are known to vary in terms of quality of service (QoS). The variations from network to network can be static such as because of the inherently different capabilities of the networks (e.g., IEEE 802.11a vs. IEEE $802.11 \mathrm{n}$ ) or they can be dynamic based on each network's current congestion level. Selection of an optimal access network for service delivery is an important problem to be solved in such an environment. Since a number of network attributes, e.g., related to QoS have an impact on this decision, the use of Multi-Attribute Decision Making (MADM) algorithms [1] has been proposed in the past for ranking candidate access networks in terms of their suitability. A comprehensive MADM approach involving application of non-compensating MADM algorithm followed by compensating MADM algorithm has been proposed in [2]. Output of these algorithms is dependent upon the accuracy of information being used as their inputs. In real world scenarios, frequently some of the information is either imprecise or not available. In addition in some cases, because of the candidate network types the usage of fuzzy input information is more useful than crisp values. Figure 1 illustrates that using MADM when all the information is not available or is not precise may not bring the selection down to one candidate network of choice. But it is still possible to narrow down the initial list of candidate networks. Below we describe scenarios where application of MADM algorithms for network selection while relying on incomplete information can be beneficial.

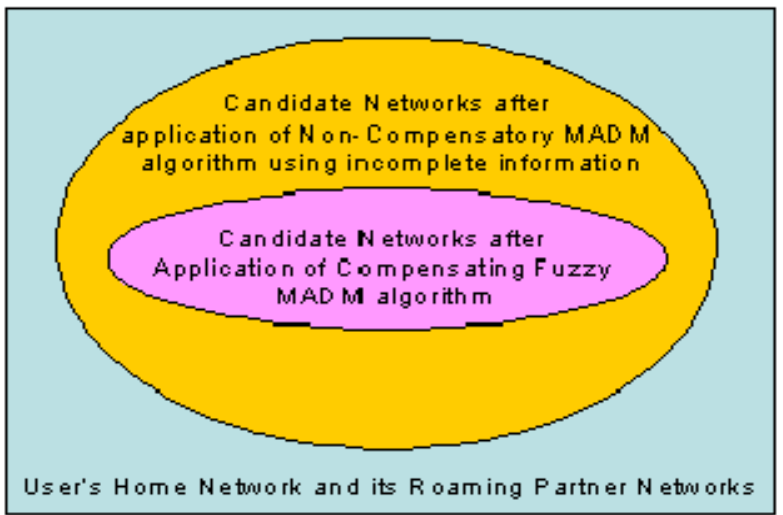

Figure 1. Use of MADM with imprecise information can help narrow down the candidate service delivery network options 


\section{USING NON-COMPENSATING MADM ALGORITHMS WITH INCOMPLETE INFORMATION}

In the network selection process described in [2], initially, noncompensating MADM algorithms are used to narrow down the candidate list. Application of a non-compensating algorithm can be described as a simple matching process of user/terminal requirements/capabilities to network capabilities, e.g., matching of wireless technologies supported by the terminal and the network. The information used in this process comes from both the terminal and the entities within the network. However in some cases the terminal or network entities may either be unable or unwilling to provide some of the information to the decision making entity. For example, the information related to terminal's mobility profile may not be available for the decision making entity because the user / terminal has not indicated it. In other cases the decision maker may not have all the information about the candidate networks that is to be used in the non-compensatory part of the MADM algorithm. For example, it may not know the exact coverage area or the authentication methods supported by the roaming partner access networks. Even in such scenarios, by using the mechanisms described in [2] candidate networks can be narrowed down to fewer and more probable service delivery networks. This is achieved by not applying the algorithm for the missing attributes. The candidate networks in the resultant shortened list in such scenarios may not all be accessible to the terminal but the list can provide guidance to the terminal to help it narrow down the service delivery options.

In general, the attributes used in the non-compensating part of the algorithm can be separated into those essential for the decision process and those that can be considered less critical. The decision maker can decide which attributes it considers absolutely essential to get useable information for the decision process. For example without the information about the location of the terminal it is not possible to narrow down the search, whereas information about service to be used can be considered less critical. Also, in such scenarios there will be a possibility that some of the top ranking networks in the short list are not accessible to the terminal, e.g., if authentication mechanism related information was lacking for the network or terminal type. Therefore, to make the information useful, a list of preferred networks instead of a single top ranking network should be made available to the terminal.

\section{USINg COMPENSATING MADM ALgoRITHMS WITH DATA PREDICTION AND FUZZY INPUT}

The application of a standard compensating MADM algorithm to network selection involves

3.1 Identifying all alternatives and compensatory MADM attributes impacting the decision process,

3.1 Assigning relative importance in the decision making process to each of the attributes, and

3.1 Using a MADM algorithm to get a ranking of the alternatives.

These algorithms are used for ranking the alternatives in terms of their desirability as a whole with respect to multiple criteria that can influence the decision. The following two scenarios have been identified where fuzzy approaches will be useful while using the compensating MADM algorithm during network selection.

\subsection{Scenario 1 - Imprecise or missing information}

For compensating MADM algorithms to be working properly in order to select the optimal service delivery network, the attribute values have to be reliable. However, as shown in Figure 2, due to the geographic distribution of the data collection points [3] it may not be possible to get real time updates for some of the attributes used in network selection. Also the figure shows that while dealing with heterogeneous access technologies spanning autonomous operator domains, it may not be possible to get a homogeneous set of attribute data spread evenly over time that would allow a direct comparison between the networks towards their suitability for delivering requested services. In many cases there is measurement error associated with monitoring and processing of dynamic QoS attribute values such as packet delay, jitter, and loss. Hence there is a need to develop a mechanism of network selection when input attribute values are less reliable or unavailable.

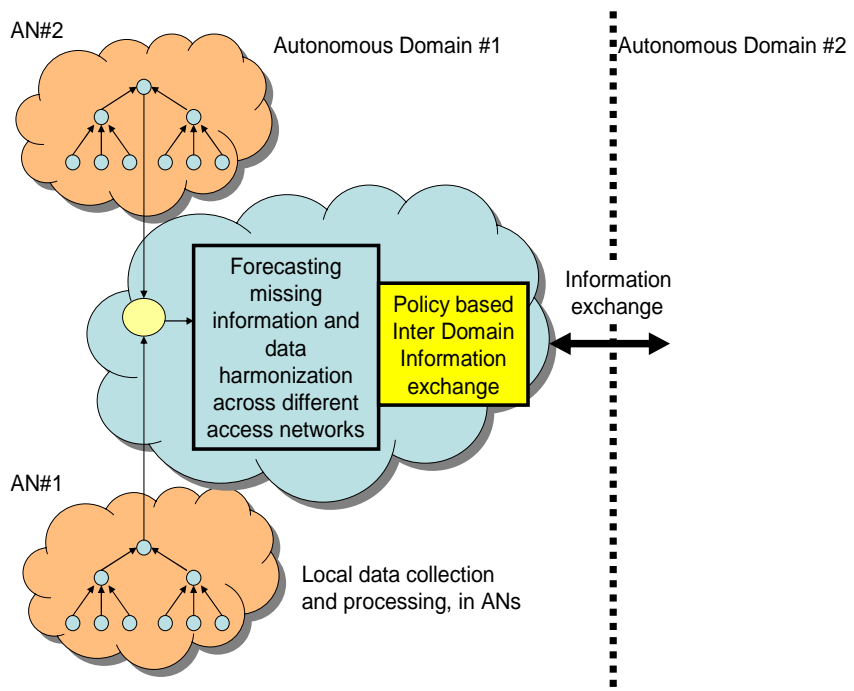

Figure 2. Distributed nature of data collection points across different access types and autonomous domains makes harmonized, accurate and real time parameter information challenging.

Zadeh [4][5] developed in the 1960s the fuzzy theory that enables algebraic manipulation of fuzzy or imprecise data. Since then fuzzy theory has found application in a variety of areas including decision support. Fuzzy numbers have been used in the past with MADM algorithms for scenarios where input attribute values are imprecise or hard to calculate [6][7]. Use of a very simple fuzzy logic based multiple-criteria decision-making system has been proposed [8] to perform vertical handovers in a heterogeneous network environment. However the paper adopts a very simplistic approach and has not taken into consideration important practical aspects such as prediction or estimation of unavailable data, selection of fuzzy number types to represent predicted attributes, suitable defuzzification techniques or even the selection of an appropriate Fuzzy MADM algorithm. The application of [8] to network selection is therefore limited. In [8] fuzzy based network selection has been discussed within the context of peer to peer networking. In [9] and [10] fuzzy mechanisms have been considered within the context of vertical handoffs. [9] actually converts fuzzy data to crisp values before applying standard MADM algorithms. [10] uses a fuzzy inference engine along with 
neural nets for prediction about number of users. [8]-[10] however have a somewhat different focus and do not take into consideration important practical aspects for the problem under consideration in our research such as data prediction for unavailable data, selection of fuzzy number type to represent predicted attributes, suitable defuzzification techniques or even a fundamental consideration about when it is appropriate to use fuzzy MADM algorithm. Here we describe a comprehensive solution to the problem by identifying scenarios where fuzzy techniques will be useful and scenarios where other mechanisms can be applied. A novel way of combining fuzzy techniques along with parameter estimation in network selection has been proposed. They have been applied to the decision process while using a proposed Fuzzy implementation of Grey Relational Analysis (GRA), a multi attribute decision making algorithm. We describe mechanisms for prediction/estimation of missing data, fuzzification of the estimated values and the subsequent defuzzification of network rankings obtained by use of Fuzzy GRA. Additional decision support tools that can be applied under such conditions have been described to indicate Confidence Levels either in the network rankings or in the data before network rankings are obtained. Both approaches have been described in this paper. Together, the techniques described here improve the reliability of the results and allow decisions to be made under uncertain conditions. The mechanisms described here would work well with the network assisted terminal based network selection architecture described in [3].

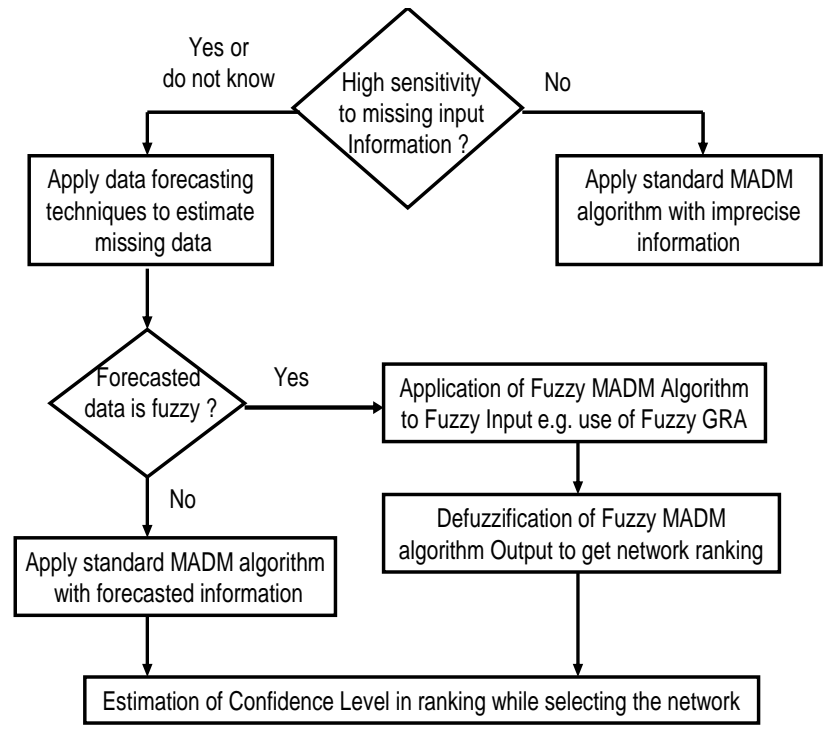

Figure 3. Steps involved in proposed network selection mechanism with imprecise information

Figure 3 shows a comprehensive approach towards network selection mechanism that leverages parameter estimation techniques, fuzzy theory, MADM algorithms and also introduces the new concept of Confidence Level. The first step is to check if the service being requested or user's subscription profile is sensitive to the attribute data that is missing. For example, a bronze subscription user or a web browsing service may not be sensitive to small variations in QoS related attribute values. This is primarily because of the low weights assigned to the QoS related attributes in the MADM decision process. If sensitivity is low, standard MADM algorithm can be applied even with imprecise attribute information. However if the sensitivity is high or sensitivity can not be determined because of, e.g., missing service / subscription related information then data estimation should be done. Depending upon the estimation methods used and the past experiences with the estimation process, the forecasted data can be expressed as scalar values or fuzzy sets. For fuzzy data values, the fuzzy implementation of MADM algorithm is then applied to get a fuzzy ranking of the networks. This is followed by the step where fuzzy rankings are defuzzified. For the case of non-fuzzy estimated values, standard MADM algorithm is applied. In the final step, any additional information to judge the Confidence Level in the network ranking obtained is taken into consideration. In another approach described later in the paper, which has some advantages to the approach in Figure 3, Confidence Level estimation is performed immediately after forecasting of missing data.

\subsubsection{Data Forecasting}

A uniform set of attributes of each candidate access network has to be provided as input to the decision algorithm to form the basis for network selection. Data prediction can be applied to forecast attribute values in the future based on past history of the data. For example, based on time of day and usage pattern, many QoS attributes such as the utilization of a hotspot can be predicted to a certain degree. Typically the observed values are represented in the form of a time series which is a collection of observations ordered in time. Deterministic data prediction can be performed using the following methods:

\subsubsection{Seasonal trends and averaged values}

Smoothing techniques can be used to identify underlying trends in the observed attribute values when some of the data to be used in the decision making process go missing. The smoothing of time series removes noise related irregularities and enhances the informational part of the observed data. Several smoothing techniques exist; e.g., Simple Moving Averages (SMA) is useful for the type of data that exhibits static values for mean and variance. SMA of order $n$ at time $t+1$ can be described by the following formula:

$$
S M A_{t+1}=\frac{\left(V_{t}+V_{t+1}+\ldots+V_{t-n+1}\right)}{n}
$$

Weighted Moving Averages (WMA) involves assigning different weights to historical data before taking the moving average. It can be represented as follows:

$$
W M A_{t+1}=\frac{\left(w_{t} * V_{t}+w_{t+1} * V_{t+1}+\ldots .+w_{t-n+1} * V_{t-n+1}\right)}{n}
$$

where

$$
\sum_{i=t}^{i=t-n+1} w_{i}=1
$$

Other types of moving averages include various forms of Exponential Smoothing (ES) techniques [13]. The use of a particular smoothing technique would depend upon its ability to accurately predict the attribute values. The prediction accuracy can be measured by running the algorithm on prior collected data and comparing the actual and the predicted values. 


\subsubsection{Regression}

Regression [13] analyzes the relationship among variables to estimate the value of one variable from known values of other variables related to it. In the case of network selection, an attribute with known value can be used in calculating another input attribute because of a strong correlation. In regression analysis trends of variables (such as linear) under consideration are analyzed and the variables that are seen to have dependencies on each other are correlated and then modeled using polynomials. The variables that can be easily measured with least error are also identified. A regression using only one predictor is called a simple regression. For example if network utilization is easy to measure and report but packet jitter, packet loss, or latency are not readily available, then these QoS related parameters can possibly be estimated by network utilization because of a correlation between them under normal network conditions.

The actual process of finding the relationship can involve sample data collection, drawing scatter plots to understand the relationship amongst the variables, and using computer packages or modeling techniques (such as linear prediction using least square method) to figure out the relationship. Typically relationship only holds for a limited range of variable values and only provides an estimate or average value.

As an example, an access network under consideration may be able to provide its network utilization more readily, reliably and on a continuous basis compared to other QoS related parameters such as packet loss, delay and jitter, which can require much more active monitoring of the network. Under normal operating conditions network utilization is correlated with QoS aspects in a packet switched network, using regression analysis as described in this section. Therefore utilization (U) that is much easier to monitor and to report on, can be related for range of values with packet Delay (D) , Jitter (J) and Loss (L). Similar relationship between network utilization and QoS parameters maybe provided by the operator of the network to its partners as part of the roaming agreements or service level agreements (SLAs).

\subsubsection{Fuzzy Estimated Values}

Because of the forecasting techniques used and other factors such as the prior experience with use of forecasted data, non crisp values may be obtained which can then be represented as Fuzzy Numbers. A Fuzzy Number [4][5] forms a fuzzy set that can have different membership grades as shown in Figure 4. A very common type of fuzzy set membership results in a Triangular Fuzzy Number (TFN). With the type of problems that can arise in data acquisition such as measuring inaccuracies or lack of updated real time attribute data as described earlier, a triangular shaped fuzzy number will be a good fit that is simple enough to be used in real time calculations. For example when regression analysis is used for data prediction, the value of the predicted parameter can be described by a triangular fuzzy number. Figure 4 also provides mathematical representation of membership function for a TFN and simple mathematical operations like addition and multiplication between two TFNs.

Use of tools such as scatter plots shows that in general the correlation defined by regression between the variables is never exact. For example, in the case of the regression scenario described earlier, at lower utilization there will not be any congestion conditions in the access network and therefore it can provide high level of QoS to all the services or all IP flows. The regression equations would provide an accurate relationship. At higher utilization the access network could distinguish between preferred and non preferred services or IP flows in the packet scheduling process. As a result, the aggregate observed data for all services/IP Flows would show a spread/scatter at higher utilization. This behavior can be represented by mapping attribute values of $D, J$ and $L$ to a range of $U$ values. For this example we consider that they map to $0.8-1.1 \mathrm{U}$ with $1.0 \mathrm{U}$ representing the default or modal value.

In fuzzy numbers terms, this can then be represented by triangular fuzzy numbers shown graphically in Figures 4 . It can be seen that the uncertainty in values for $\mathrm{D}, \mathrm{J}$ and $\mathrm{L}$ increases with an increase in network utilization (U). Fuzzy number sets can also be obtained by direct mapping from lookup tables that map a known value of $\mathrm{U}$ to $\mathrm{D}, \mathrm{J}, \mathrm{L}$ values observed earlier for the same value of $\mathrm{U}$ in that network (as described by seasonal tends in previous section).
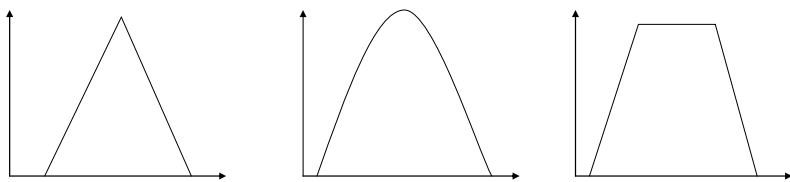

Simple Fuzzy Number Sets

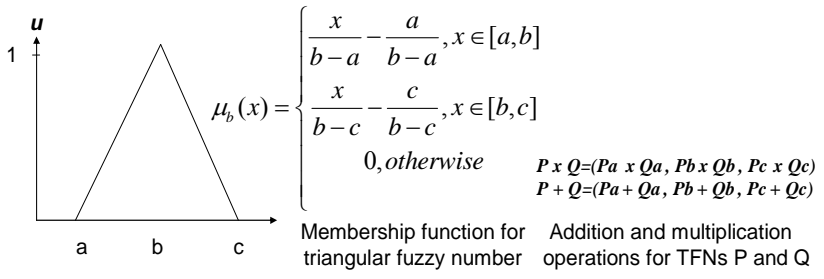

Triangular Fuzzy Number (TFN)

Figure 4 Fuzzy Numbers

\subsubsection{Compensating MADM algorithm}

MADM algorithms [1][15] include a variety of deterministic mechanisms that have been used in decision making for a range of engineering problems. Fuzzy logic can be used in conjunction with MADM algorithm. Triantaphyllou in [6] and [7] has described the use of fuzzy information with some of the commonly used MADM algorithms.

Depending upon the type of ambiguity in the decision process, fuzzy logic can be applied in more than one ways with MADM; e.g., the uncertainty in the decision process can be in the attribute values or in the importance associated to them. Here, it is assumed that the importance or weight assigned to an attribute is based on the service requested or the user's QoS subscription and therefore known based on the information provided by the user. However, because of the reasons described earlier in the paper, some of the input attribute values are either unavailable or are imprecise.

Here Grey Relational Analysis (GRA) [12][14] is used as a MADM algorithm for fuzzy implementation described in this paper. Grey Relational Analysis (GRA) [14] is based on the concept of Grey Relational Space (GRS). GRS (X,Y) describes a relationship $Y$ between reference data values $X 0$ and sequence of data values $X$. So if $y \in Y, \quad X, X_{i}, X_{0} \in X_{0}$, such that

$x_{0}=x_{0}(1), \ldots \ldots x_{0}(n)$ and $x_{i}=x_{i}(1), \ldots \ldots . x_{i}(n)$ then $y\left(x_{0}(k), x_{i}(k)\right)$ 
would represent a GRS at point $\mathrm{k}$ provided the axioms documented in [14] are satisfied. GRA calculates Grey Relation Coefficient (GRC) and uses it as a measure of the closeness of the result to the reference values. For the problem of network selection, the value of GRC provides a measure of ranking of the networks.

While using fuzzy implementation of GRA, for the scenarios under consideration the attribute values to be used as input to the algorithm will be a mixture of crisp and fuzzy values. This is because not all of the attribute values are unknown or have imprecise values. For networks with fuzzy input attributes represented by a TFN, it would result in three GRCs to be evaluated using the fuzzy arithmetic for TFN as shown in Figure 4. This assumes that because of correlation amongst missing input attribute values, multiple fuzzy inputs described by TFNs will be equivalent to a single triangular fuzzy input. A defuzzification process is then used to get a crisp value of GRA coefficient.
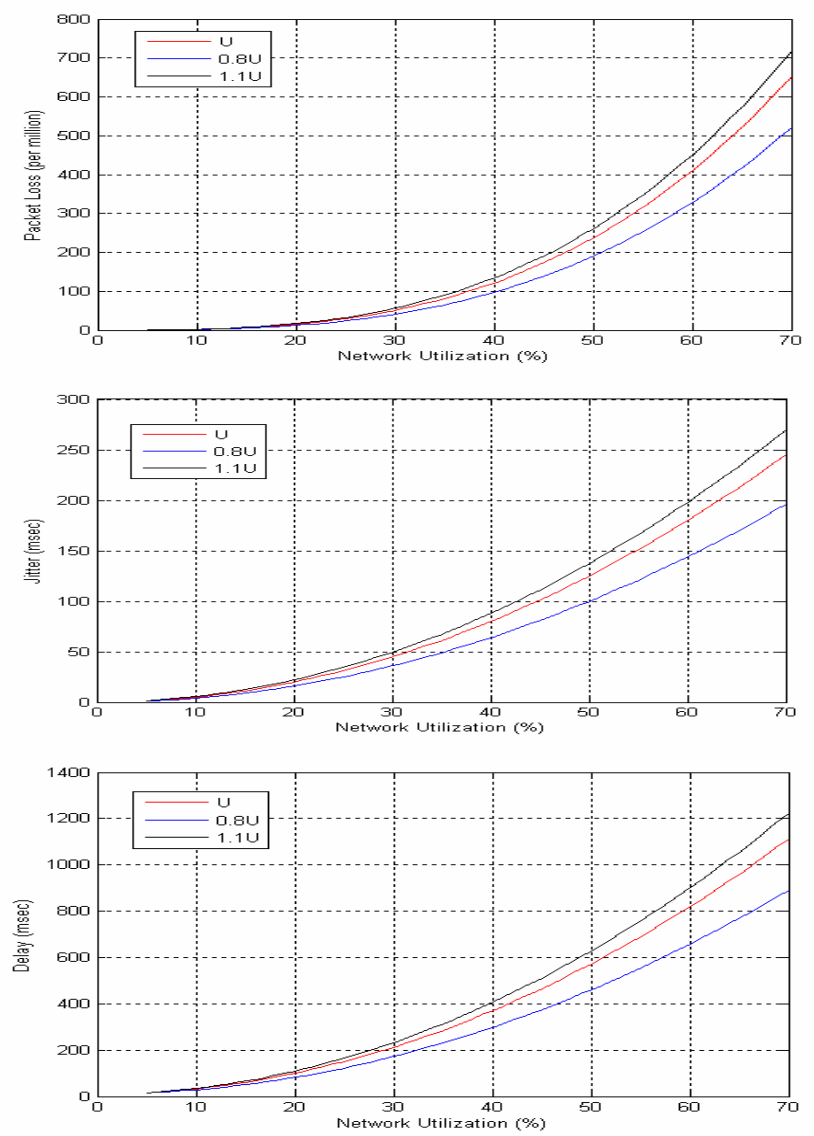

Figure 5. Example graphs of packet Delay (D), Jitter (J) and Latency $(\mathrm{L})$ values using Network Utilization (U). It assumes corelation represented by $D=0.225 * U^{2}+10, J=0.05 * U^{2}$, $L=0.0019 * U^{3}$ with $\mathbf{0 . 8 - 1 . 1} \mathrm{U}$ in the network utilization range of $5 \%$ and $70 \%$

In order to better understand the process illustrated in Figure 3 we consider a simple scenario where network selection decision has to be made with five network options. These networks are represented by a set of QoS related attributes as listed in Table I. The selection of these attributes for use in network selection while using MADM is described in [2][18][19]. Each attribute has a relative importance represented by a weight assigned to it during the decision process, which can be based on the service being requested or the QoS profile of the user. The weights shown in Table I represent importance assigned to the attributes for a streaming service and web browsing. We have selected streaming service as a representative service scenario that is sensitive to dynamic QoS attributes and web browsing as a representative service scenario that is not sensitive to dynamic QoS attributes.

Table I. Attributes and their weight assignments for streaming media and web browsing services when using Fuzzy GRA

\begin{tabular}{|c|c|c|}
\hline Attribute & $\begin{array}{c}\text { Stremaing } \\
\text { Media }\end{array}$ & $\begin{array}{c}\text { Web } \\
\text { Browsing }\end{array}$ \\
\hline $\begin{array}{c}\text { Cost per Byte (CB) } \\
\text { Data transport cost on a particular access system }\end{array}$ & 0.2 & 0.5 \\
\hline $\begin{array}{c}\text { Total Bandwidth (TB) } \\
\text { Overall bandwidth of the wireless access link }\end{array}$ & 0.15 & 0.05 \\
\hline $\begin{array}{c}\text { Allowed Bandwidth (AB) } \\
\text { Bandwidth per user allowed by the access system }\end{array}$ & 0.2 & 0.15 \\
\hline $\begin{array}{c}\text { Utilization (U) } \\
\text { Current utilization of the wireless link }\end{array}$ & 0.2 & 0.1 \\
\hline $\begin{array}{c}\text { Packet delay (D) } \\
\text { Average packet delay within the access system }\end{array}$ & 0.1 & 0.05 \\
\hline $\begin{array}{c}\text { Packet Jitter (J) } \\
\text { Average packet delay variations within the access } \\
\text { system }\end{array}$ & 0.1 & 0.05 \\
\hline $\begin{array}{c}\text { Packet Loss (L) } \\
\text { Average packet loss rate within the access system }\end{array}$ & 0.05 & 0.1 \\
\hline
\end{tabular}

Table II lists the values for these attributes for Ntwk\#1 through Ntwk\#4 at the instance of decision making. QoS related data (i.e., Delay, Jitter and Packet Loss) for Ntwk\#5, one of the networks under consideration, are not available at the time of decision making. However, the last known reliable value for these attributes for Ntwk \#5 is available and are listed in Table II. We assume that based on prior observations of the network, it has been possible to correlate network utilization with QoS parameters. The correlation used in this example is defined in Figure 5. It results in TFNs for Delay, Jitter and Latency values for Ntwk\#5 as shown in Table II.

Table II. Attribute values for the five networks under consideration

\begin{tabular}{|c|c|c|c|c|c|c|c|}
\hline & $\begin{array}{c}\text { CB } \\
\text { \% }\end{array}$ & $\begin{array}{c}\text { TB } \\
\text { Mbps }\end{array}$ & $\begin{array}{c}\text { AB } \\
\text { Mbps }\end{array}$ & $\begin{array}{c}\text { U } \\
\text { \% }\end{array}$ & $\begin{array}{c}\text { D } \\
\text { msecs }\end{array}$ & $\begin{array}{c}\text { J } \\
\text { msecs }\end{array}$ & $\begin{array}{c}\text { L } \\
\text { per 10 }\end{array}$ \\
\hline Ntwk\#1 & 100 & 2 & 0.2 & 10 & 400 & 50 & 100 \\
\hline Ntwk\#2 & 20 & 11 & 1 & 20 & 200 & 25 & 20 \\
\hline Ntwk\#3 & 10 & 54 & 2 & 20 & 100 & 15 & 15 \\
\hline Ntwk\#4 & 5 & 100 & 5 & 40 & 150 & 30 & 20 \\
\hline Ntwk\#5 & 30 & 100 & 5 & $20^{*}$ & $100^{*}$ & $20^{*}$ & $15^{*}$ \\
& & & & $25^{\prime}$ & $122^{\prime}$ & $25^{\prime}$ & $24^{\prime}$ \\
& & & & & $151^{\prime}$ & $31^{\prime}$ & $30^{\prime}$ \\
& & & & & $165^{\prime}$ & $34^{\prime}$ & $33^{\prime}$ \\
\hline
\end{tabular}

*- last reliable attribute values

'- Forecasted attribute value represented as a Triangular Fuzzy Number 
Using the fuzzy version of GRA described in the previous section for streaming media service, the results as shown in Figure 6 are obtained. It can be seen that for Ntwk\#5, three GRC values are obtained which constitute a GRC represented by a TFN. With Ntwk\#5 represented by a Fuzzy GRC, the exact ranking for the networks is not obvious and therefore a defuzzification step is required. Note that although in this example changing the attribute values for one network, in this case Ntwk\#5, does not impact the GRC values for the other networks, this is not always the case. In most of the cases, due to the way GRC is calculated, different GRC values may also result for some of the other alternative networks. In that case the defuzzfication part can become more complicated as we will have to apply defuzzification techniques to other network alternatives as well in order to obtain crisp rankings. This would become clearer in the example in the next section.

\subsubsection{Defuzzification}

Defuzzification is the process of converting a fuzzy number or set to a crisp value. Several mechanisms exist for comparing fuzzy numbers with some of them fairly complex and computationally intensive. A good mechanism can be selected by considering its level of complexity, its accuracy and its application to fuzzy numbers of a particular shape. In our simple example we assume that the output of GRC with TFN input is also a TFN. This assumption can not be generalized and more complex cases would need further analysis about the expected shape of the output fuzzy number. A range of defuzzification techniques are documented in [16][17]. Center of gravity $(\mathrm{CoG})$ is one of the computationally simpler and more popular technique that is particularly well suited for defuzzification of TFN. It calculates the centroid of the fuzzy values and can be approximated in the discrete domain as follows.

$$
C o G \approx \frac{\sum_{j=1}^{r} \mu_{j} * F_{j}}{\sum_{j=1}^{r} \mu_{j}}
$$

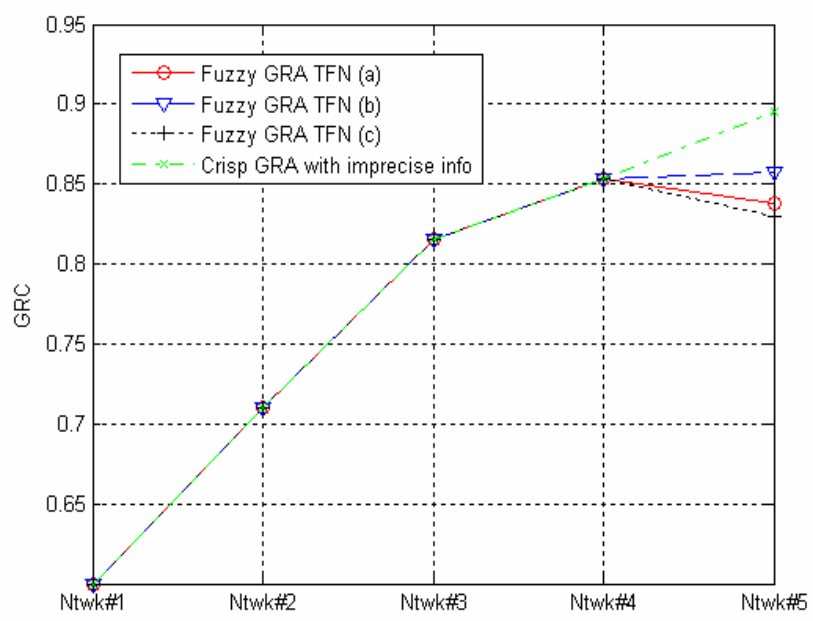

Figure 6. Results for streaming media services using GRA algorithm with fuzzy input and with imprecise input information where $\mu_{j}$ and $F_{j}$ represent the fuzzy set membership index and the corresponding fuzzy number, respectively, in the discrete domain.

Figure 7 graphically shows the application of $\mathrm{CoG}$ for defuzzification of GRC values for Ntwk\#5 obtained from Figure 6. Comparing the $\mathrm{CoG}$ value for Ntwk\#5 from Figure 7 with results for the remaining networks shown in Figure 6 it can be seen that the CoG based GRC value for Ntwk\#5 is lower than that for Ntwk\#4. Hence Ntwk\#4 should be selected.

In the absence of reliable data for some of the networks, the alternatives to not using the techniques described above would be to

- Apply standard MADM technique for all the networks without fuzzification and use instead non-fuzzy unreliable data or its approximation as input.

- Remove the networks with unreliable or missing data altogether from the comparison and then perform MADM algorithm on the remaining to obtain their rankings.

If the first approach is used, depending upon the error in the data and the sensitivity of the service/user subscription to it there is a possibility of getting an incorrect ranking of the networks because of unreliable input to the algorithm. For streaming media service this is shown in Figure 6 where the last reliable values of the missing parameters were used. However the values were outdated and since then network utilization had gone up. So because of a strong correlation between network utilization and the missing QoS attributes, the values of the missing attributes could have been predicted since streaming media service is sensitive to the QoS attribute values. The use of outdated values in this case leads to the selection of Netwk\#5 whereas Ntwk\#4 would have been a better choice as shown by the use of Fuzzy GRA also illustrated in Figure 6.

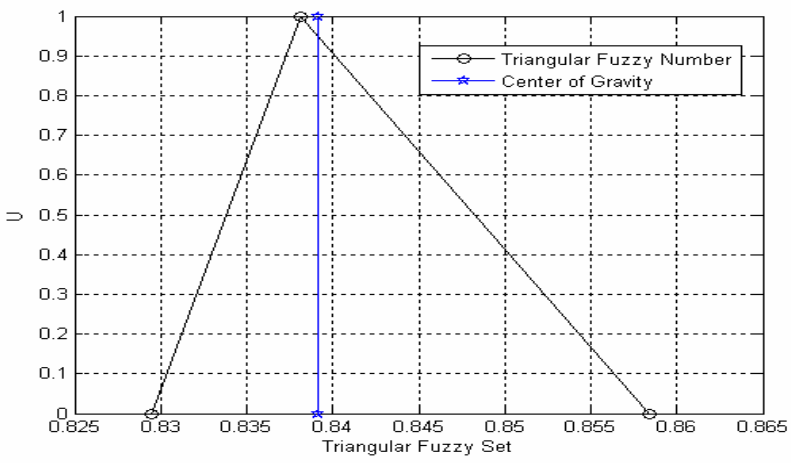

Figure 7. Use of Center of Gravity (CoG) for defuzzification of a TFN representing GRC in fuzzy implementation of GRA for streaming media services

If the second approach is used, it is possible that a top ranking candidate network is dropped before the comparison and hence not selected. For the same example described above, the results of applying GRA after removing Ntwk\#5 from the candidate list are shown in Figure 8. In the example, the GRC values and hence the ranking of the remaining networks is unchanged. While in this case Ntwk\#4 is correctly selected in the given example, had the network utilization gone down instead then it was entirely possible that Ntwk\#5 would have been a better choice but it would have been eliminated as a candidate by this strategy. 
Now we consider the case of web browsing service. In the case of this service, based on the weights assigned to the attributes the sensitivity of the service to dynamic QoS attributes is known to be low. In order to confirm our assertion that for this type of service the use of imprecise information is acceptable, we apply both Fuzzy (with parameter estimation) and crisp versions of GRA algorithm. The results of Fuzzy GRA algorithm with forecasted data documented in Table II are shown in Figure 9. The results of defuzzification are shown in Figure 10. The results of application of crisp GRA while using imprecise information for missing attributes are shown also in Figure 9. Comparison of the two results shows that Ntwk\#4 gets selected in both cases by a wide margin and the use of imprecise information does not have much impact on the results.

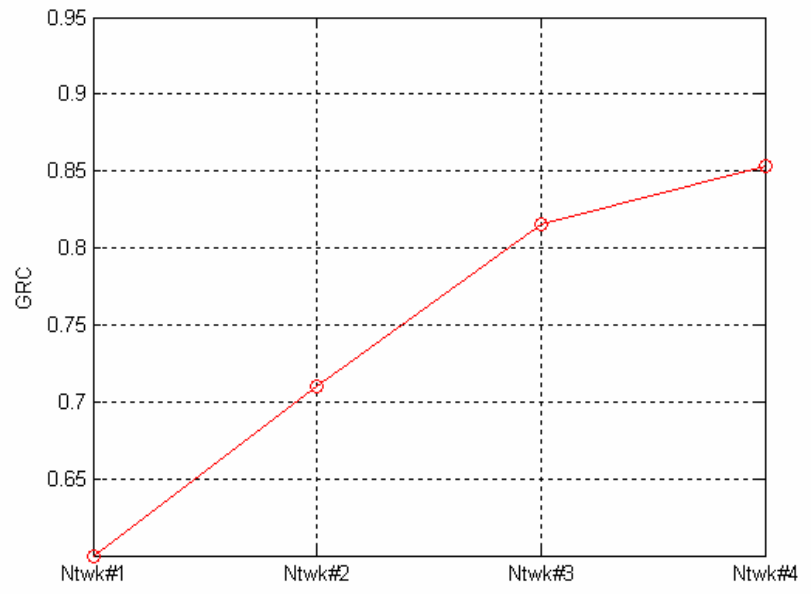

Figure 8. Results of GRA algorithm for streaming media service after removing Ntwk\#5 with missing information

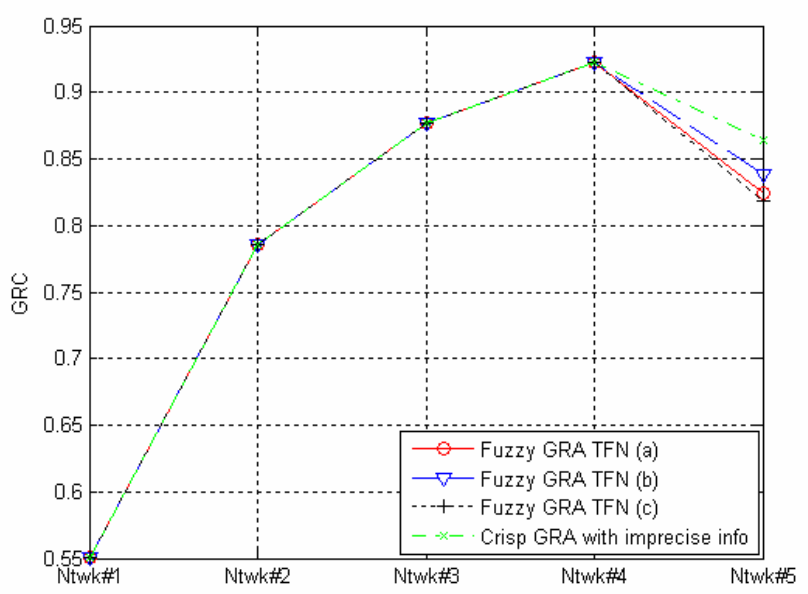

Figure 9. Results of for web browsing service using GRA algorithm with fuzzy input information and imprecise input information
An appropriate use of parameter estimation and the fuzzy techniques as described in Figure 3 hence provide a balanced approach by not eliminating the networks with missing, old or unreliable data while keeping in view the fact that the information about them is fuzzy and/or not entirely accurate. In the following section the proposed mechanism has been further enhanced by developing criteria for judging the reliability of the results.

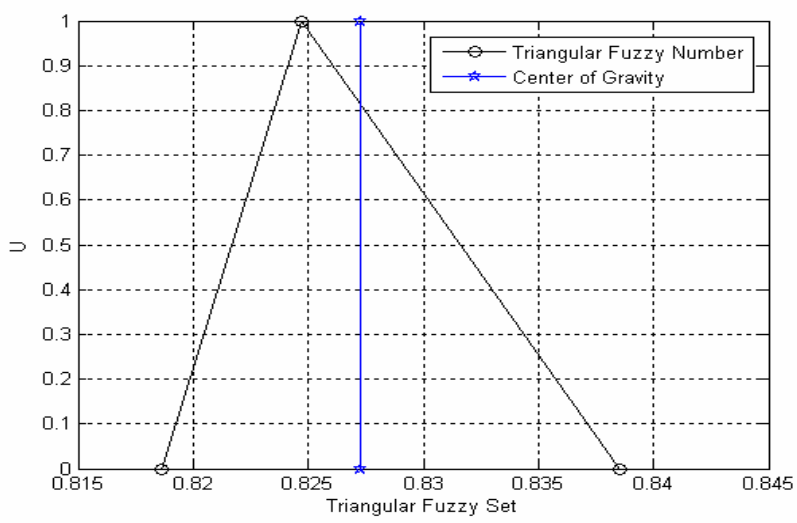

Figure 10. Use of Center of Gravity (CoG) for defuzzification of a TFN representing GRC in fuzzy implementation of GRA for web browsing services

\subsubsection{Confidence Level}

Although methods described above can provide improved ranking for the networks under consideration, other factors should also be taken into consideration before making use of rankings obtained in this manner. Here we introduce a new concept of "Confidence Level" that can allow the decision maker to evaluate if the network rankings are reliable enough to be used for network selection. The factors used in calculating Confidence Level include the followings.

Sensitivity of service or user subscription type to unreliable attribute values $\left(C L_{s}\right)$ : As described earlier, in applications of MADM processes, attribute weights are assigned based on the service or user type; e.g., a VoIP service or a gold user may be assigned higher weights for dynamic QoS related attributes because of the nature of the requested service or the user subscription. Based on the weights assigned to attribute values, network ranking can become more vulnerable to errors in parameter estimations. In other words decisions where dynamic QoS attributes have higher assigned weights will have higher sensitivity to errors in parameter values. For example a VoIP service when compared to web browsing is more vulnerable to incorrect ranking because of imprecise values of QoS related attribute. This is because of the relatively higher weights assigned to dynamic QoS attributes such as packet delay, jitter, loss, etc., in decision making for QoS sensitive service types.

Time since last data update $\left(C L_{t}\right)$ : There will be a level of uncertainty in the data value based on how long data has been unavailable and how much it can change over time based on, e.g., seasonal charts. Its value would indicate that the older the last good value, the lesser the Confidence Level in its estimated value. 
The degree of correlation with attribute whose value is known and is used in regression analysis $\left(C L_{c}\right)$ : This indicates the level of confidence in the estimated value. A strong correlation would indicate for example a high Confidence Level.

We define a minimum threshold ( $\left.\mathrm{CL}_{\text {threshold }}\right)$ of Confidence Level (CL) for acceptable level of ranking results. In other words, for the ranking to be used, the following should be true.

$$
C L>C L_{\text {threshold }}
$$

The value of the Confidence Level threshold $\mathrm{CL}_{\text {threshold }}$ can be provisioned into the process by the decision maker based on decision policies. In the case of Confidence Level being lower than the threshold, the decision maker will remove the network with imprecise information from the list of alternatives. However it is known [7] that addition or removal of an alternative during MADM algorithm application can impact the ranking for the rest of the alternatives non-uniformly. It may therefore be necessary to run the algorithm again in this situation. An alternative approach can be to calculate the Confidence Level before using the MADM algorithm. If the Confidence Level is found to be below the threshold for all alternatives with imprecise information, then all alternatives with imprecise information are removed from comparison and a standard MADM algorithm is applied to the rest. Otherwise only those alternatives with Confidence Level lower than the threshold are removed and the fuzzy version of the MADM algorithm is applied to the rest. This approach is shown in Figure 11.

Using the alternative approach and the factors described above, the decision maker can formulate an overall Confidence Level to be used in the decision process. A very simple example of formulating such a term can be

$$
\mathrm{CL}=\mathrm{w}_{\mathrm{s}} * \mathrm{CL}_{\mathrm{s}}+\mathrm{w}_{\mathrm{t}}{ }^{*} \mathrm{CL}_{\mathrm{t}}+\mathrm{w}_{\mathrm{c}} * \mathrm{CL}_{\mathrm{c}}
$$

where $0 \leq C L \leq 1$. and $\sum w_{i}=1$

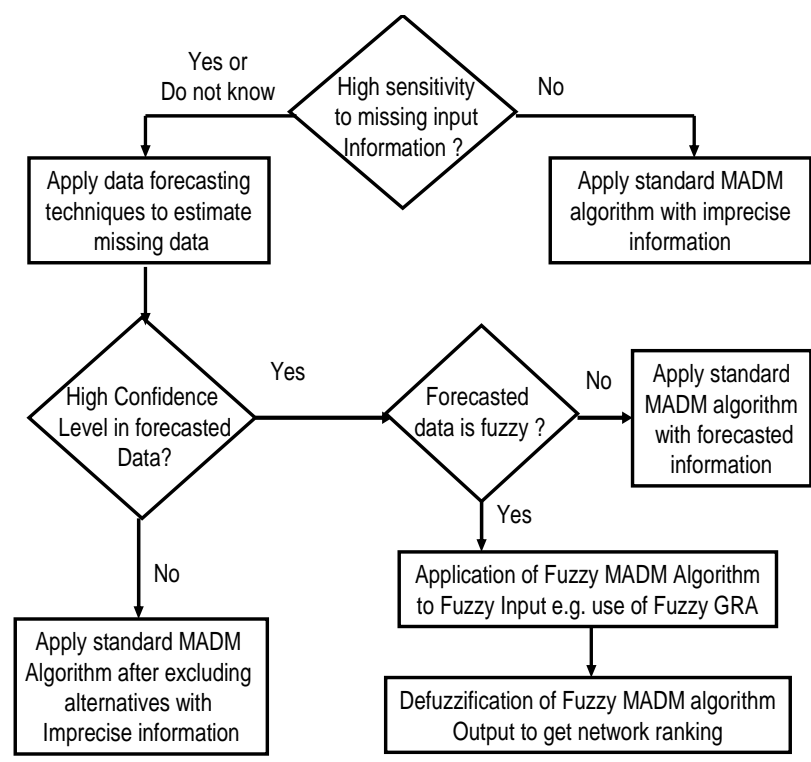

Figure 11. Alternative approach of using Confidence Level in decision process
The decision maker can also provide, based on his understanding of how various factors should influence Confidence Level, some distribution of weight across $\mathrm{CL}_{\mathrm{s}}, \mathrm{CL}_{\mathrm{t}}$, and $\mathrm{CL}_{\mathrm{c}}$. Here we use an equal distribution of weights between the three (i.e. $\mathrm{w}_{\mathrm{s}}=\mathrm{w}_{\mathrm{t}}=$ $\mathrm{w}_{\mathrm{c}}=0.33$ ) so that $0 \leq \mathrm{w}_{\mathrm{s}} * \mathrm{CL}_{\mathrm{s}} \leq 0.33,0 \leq \mathrm{w}_{\mathrm{t}} * \mathrm{CL}_{\mathrm{t}} \leq 0.33$ and $0 \leq$ $\mathrm{w}_{\mathrm{c}} * \mathrm{CL}_{\mathrm{c}} \leq 0.33$. Using the concepts described earlier and assuming a linear relationship between the variables and utilizing normalized values, we come up with the following two equations:

$$
C L_{t}=-0.33 t+0.33
$$

where $\mathrm{t}$ is normalized time; i.e., $0 \leq \mathrm{t} \leq 1$. with $\mathrm{t}=1$ representing a finite elapsed time since the last reliable value after which data values can no longer be considered useful..

$$
C L_{c}=0.33 \mathrm{c}
$$

where "c" is a normalized correlation factor; i.e., $0 \leq \mathrm{c} \leq 1$, which is determined by the decision maker based on past data collection.

Similarly we can relate $\mathrm{CL}_{\mathrm{s}}$ to normalized value of sensitivity factor determined by the decision maker as follows

$$
C L_{d}=0.33 \mathrm{~s}
$$

where $0 \leq \mathrm{s} \leq 1$.

Using these values for $\mathrm{CL}_{\mathrm{s}}, \mathrm{CL}_{\mathrm{t}}$, and $\mathrm{CL}_{\mathrm{c}}$ we come up with a simple equation for $\mathrm{CL}$ as follows

$$
C L=\frac{(s+c-t+1)}{3}
$$

where $\mathrm{s}, \mathrm{c}$, and $\mathrm{t}$ are all normalized values.

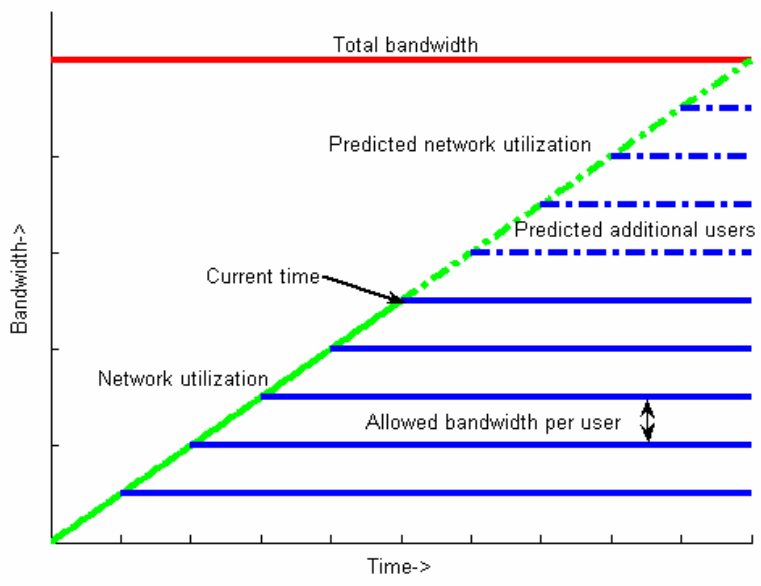

Figure 12. Graphical representation of how network congestion during service delivery time can be related to parameters such as the total bandwidth, bandwidth allowed per user, current network utilization and rate of change of network utilization.

In addition, deduction from information available about other attributes can help the final network selection process. One such example is shown in Figure 12 where the possibility of network congestion during service delivery can be predicted based on service related information of the network under consideration, 
such as the available bandwidth and total bandwidth, the current network utilization, and its past rate of change. Such information can then be used in the final selection process.

Since forecasting of data and Confidence Level in the estimated values can be done independently of the use of Fuzzy MADM algorithm, it is also possible for the decision maker to have Confidence Level in the estimated values being updated on a regular basis and being made available to the decision process when needed. A lookup table for each of the factors impacting Confidence Level can be created to map normalized values of these factors to a Confidence Level for easy calculation. A variety of decision types, from a rough estimate to very accurate, can be supported depending upon the resolution or frequency of update of the data that is being forecasted. In order to better understand the notion of Confidence Level we consider the use of lookup tables as illustrated in Table III based on the example described previously in this paper. The values used in the table are an example and will be decided in practice by the decision maker such as the network operator, based on the decision policies and the past experience with data prediction. For example, table entries for $\mathrm{CL}_{\mathrm{s}}$ indicate the fact that an unreliable Packet Jitter value will results in lower Confidence Level for VoIP type service when compared with Web Browsing. Also in Table III, it is assumed that $\mathrm{CL}_{t}$ and $\mathrm{CL}_{\mathrm{c}}$ values for Packet Delay, Jitter and Loss will be the same as these parameters are very closely related.

We set the threshold for the Confidence Level at 0.5. We assume that at the time of decision, the attribute values had not been updated for the past 5 minutes. Table III shows that in this case it has been determined that data values are not useful beyond 600 seconds i.e. 10 minutes. This would therefore give us a normalized value of $t=0.5$. Since the service type in the example is streaming media, the normalized value of $\mathrm{s}$ will be 0.75 . Also per Table III, the normalized correlation factor $\mathrm{c}$ for missing data is 0.9 . Using these values in prior equation we come up with value for CL of 0.717 which is higher than the threshold and therefore allows for inclusion of the access type in the network selection process. This type of simple calculations can help improve the reliability of results by incorporating decision maker's judgment under less certain conditions.

Table III. Example of Lookup Tables used by the decision maker for Confidence Level calculations

\begin{tabular}{|c|c|}
\hline Service Type & CL(s) for Packet Delay, Jitter and Loss \\
\hline VoIP & 0.5 \\
\hline Steaming Media & 0.75 \\
\hline Web Browsing & 1 \\
\hline
\end{tabular}

\begin{tabular}{|c|c|}
\hline $\begin{array}{c}\text { Time since last Reading } \\
\text { (sec.) }\end{array}$ & $\begin{array}{c}\text { CL(t) for Packet Delay, Jitter and } \\
\text { Loss }\end{array}$ \\
\hline 0 & 1 \\
\hline 600 & 0 \\
\hline
\end{tabular}

\begin{tabular}{|c|c|}
\hline Attribute & CL(c) with Network Utilization (U) \\
\hline Packet Delay (D) & 0.9 \\
\hline Packet Jitter (J) & 0.9 \\
\hline Packet Loss (L) & 0.9 \\
\hline
\end{tabular}

\subsection{Scenario 2 - Network types with non crisp attributes}

Some of the candidate networks may have a range of QoS values instead of one crisp value. This can be for different reasons

- A network may support multiple QoS classes or SLAs with different cost structures associated with them as described in [2]. These QoS classes / SLAs can be treated as separate alternatives while using compensating MADM algorithms. The less expensive QoS classes / SLAs may have more variability on the required QoS attributes and such variability can be represented by a nominal value with a possible spread of values around it as represented by a TFN described earlier.

- The access technologies being used by some candidate networks may be inherently incapable of providing strict QoS guarantees. This can also be based on the decision maker's prior experience with a operator network or access technology. The variability of this type can be represented by a fuzzy number.

If the service or subscription information is considered sensitive to the non crisp attribute values then network selection in such a situation can make use of Fuzzy MADM Algorithm as described in the previous section. Steps related to data harmonization, fuzzification and estimation of Confidence Level would not be required. The fuzzified input data is assumed to be available in this case (e.g., pre provisioned) and after application of Fuzzy MADM the results are defuzzified to get crisp rankings.

In order to better understand this usage of Fuzzy MADM algorithm we consider a network selection scenario [2] where multiple QoS/SLAs with a network operator constitute the alternatives. Further we assume that the user plans to use streaming media service with the attribute weights as shown in Table I. The attribute values for the QoS/SLAs classes are shown in Table IV. One of the less expensive alternatives, Ntwk\#3 as shown in Table IV, does not provide crisp QoS attribute which indicates that there can be significant variation in the values during a an ongoing session depending upon congestion levels.

Table IV. Attribute values for network alternatives under consideration

\begin{tabular}{|l|c|c|c|c|c|c|c|}
\hline & $\begin{array}{c}\text { CB } \\
\text { \% }\end{array}$ & $\begin{array}{c}\text { TB } \\
\text { Mbps }\end{array}$ & $\begin{array}{c}\text { AB } \\
\text { Mbps }\end{array}$ & $\begin{array}{c}\text { U } \\
\text { \% }\end{array}$ & $\begin{array}{c}\text { D } \\
\text { msecs }\end{array}$ & $\begin{array}{c}\text { J } \\
\text { msecs }\end{array}$ & $\begin{array}{c}\text { L } \\
\text { per 10 }\end{array}$ \\
\hline QoS Class\#1 & 10 & 100 & 0.5 & 30 & 400 & 100 & 50 \\
\hline QoS Class\#2 & 20 & 100 & 1 & 20 & 200 & 100 & 100 \\
& & & & & 75 & 25 & 25 \\
& & & & & 30 & 10 & 10 \\
\hline QoS Class\#3 & 30 & 100 & 1 & 20 & 100 & 25 & 50 \\
\hline QoS Class\#4 & 50 & 100 & 2 & 40 & 50 & 10 & 10 \\
\hline QoS Class\#5 & 60 & 100 & 5 & 60 & 40 & 10 & 10 \\
\hline
\end{tabular}

Applying the Fuzzy GRA algorithm using described earlier we get the results shown in Figure 13. As was discussed earlier, a change in attribute values for one of the networks can change the values of the GRC for other networks as well. This is shown in results of Figure 13. In order to get crisp values in this case, defuzzification 
has to be applied to GRC values of all the network alternatives. The results of defuzzification process are also shown in Figure 13, which indicate that Ntwk\#3 has the maximum GRC value and is the preferred network in this case.

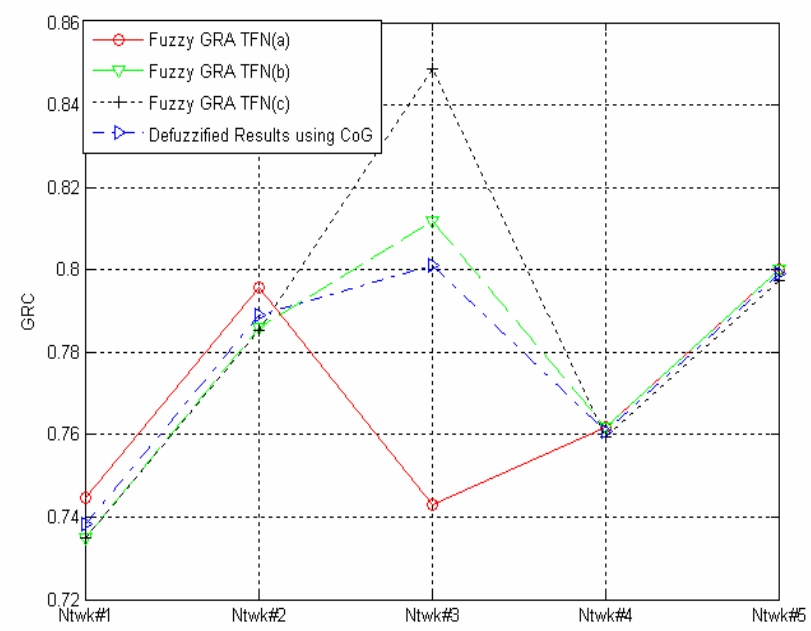

Figure 13. Results of Fuzzy GRA algorithm

The examples using fuzzy MADM described here are simple and allow us to assume that the output of MADM algorithm with TFN type fuzzy input attributes can be estimated to be also a TFN. For more complex fuzzy problems this assumption may not hold and it is an area for future research.

\section{ConClusion}

Selection of an optimal service delivery network is an important problem to be solved in an all IP heterogeneous wireless network environment spanning multiple operator domains. The problem requires special consideration when attribute related data are unreliable or unavailable. Prior studies in this area have limited applicability as they have not taken into consideration important aspects such as the sensitivity of the network selection to imprecise information, data prediction for unavailable data, use of fuzzy number type to represent predicted attributes and a decision strategy as to when to apply Fuzzy MADM algorithm. In the absence of a well defined strategy to handle scenarios described in the paper, suboptimal networks could be selected. We have provided a comprehensive network selection mechanism for scenarios with imprecise attribute information. The paper has described scenarios where fuzzy techniques along with data prediction can be applied in network selection decision process while using a Fuzzy implementation of Grey Relational Analysis (GRA), an MADM algorithm. It has described additional decision support techniques that can be applied under such conditions. These techniques have been used to develop Confidence Level in the network rankings obtained via the use of fuzzy logic and data prediction with MADM algorithm. An alternative approach for removing low confidence alternative earlier on from the decision process has also been described. The techniques described here can be tuned to address different decision type and the resolution of the data. Through examples we have shown that the techniques proposed in this paper can help improve the reliability of the results and allow for improved network selection under uncertain conditions.

\section{REFERENCES}

[1] K. Yoon and C.L. Hwang, Multiple Attribute Decision Making: An Introduction. Sage Publications, 1995.

[2] F. Bari and V. Leung, "Automated Network Selection in A Heterogeneous Wireless Network Environment", IEEE Network, January 2007.

[3] F. Bari and V. Leung, "Service delivery over heterogeneous wireless networks: network selecton sspects", in Proceedings of NGMN / IWCMC Conference, July 2006.

[4] I.A. Zadeh, "Fuzzy Sets", Information and Control, Vol. 8, pp. 338-353, 1965.

[5] I.A. Zadeh, "Fuzzy Algorithms", Information and Control, Vol. 12, pp 94-102, 1968.

[6] E. Triantaphyllou and C. Lin, "Development and Evaluation of Five Fuzzy Multi-Attribute Decision-Making Methods," Approximate Reasoning, Vol. 14, No. 4, 1996.

[7] E. Triantaphyllou, Multi-Criteria Decision Making Methods: A Comparative Study. Kluwer Academic Publishers, 2002

[8] S. Hongvan, H. Chen and J. Lingge, "Intelligent signal processing of mobility management for heterogeneous networks", in Proceedings of International Conference on Neural Networks and Signal Processing, Nanjing, China, December 2003.

[9] S. Kher; A.K. Somani, and R. Gupta, "Network selection using fuzzy logic"; Proc. $2^{\text {nd }}$ International Conference on Broadband Networks, pp.:876 - 885,Oct. 2005

[10] W. Zhang, "Handover decision using fuzzy MADM in heterogeneous networks", Proc. IEEE WCNC 2004, pp. $653-$ 658, March 2004

[11] Q. Guo; J. Zhu and X. Xu, "An adaptive multi-criteria vertical handoff decision algorithm for radio heterogeneous network", Proc. IEEE ICC 2005, pp. 2769-2773, May 2005

[12] Q. Song and A. Jamalipour, "Quality of Service Provisioning in Wireless LAN/UMTS Integrated Systems using Analytic Hierarchy Process and Grey Relational Analysis", in Proceedings of Global Telecommunications Conference Workshops, 2004. GlobeCom Workshops 2004, IEEE, 29 Nov.-3 Dec. 2004

[13] B.L. Bowerman and R.T. O'Connell, Forecasting and Time Series : An Applied Approach, 3rd Edition, Duxbury Classic Series, 2000.

[14] J.L. Deng, "Introduction to Grey System", J. Grey System, 1989.

[15] C.L. Hwang and K. Yoon, Multiple Attribute Decision Making: Mehtods and Applications. Springer Verlag, New York, NY 1981.

[16] S.M. Baas and H. Kwakernaak, "Rating and ranking of multiple-aspect alternatives using fuzzy sets", Automatica, Vol. 13, p. 47-58, Pergamon Press, 1977.

[17] G. Bortolan and R. Degani, "A review of some methods for ranking fuzzy subsets", Fuzzy sets ans systems, Vol. 15, pages 1-19, 1985.

[18] F. Bari and V. Leung, "Multi-Attribute Network Selection by Iterative TOPSIS for Heterogeneous Wireless Access", in Proceedings of IEEE CCNC Conference, January 2007.

[19] F. Bari and V. Leung, "Use of Non-monotonic Utiltiy in Multi-attribute Network Selection", in Proceedings of WirelessTelecommunication Symposium, April 2007. 